\title{
Dasselbe anders
}

\section{Repetitives Erzählen vom Sterben in Sibylle Lewitscharoffs Blumenberg (2011)}

Andreas Mauz

\begin{abstract}
"Es ist die vornehmste Aufgabe der Literatur, Totenwache zu halten und Totengespräche zu führen.»

Sibylle Lewitscharoff, Mit den Toten sprechen ${ }^{1}$
\end{abstract}

\section{Totenwache halten}

Sibylle Lewitscharoffs Blumenberg (2011) ${ }^{2}$ bietet sich aus wenigstens drei Gründen an für eine Exploration der Eigenarten literarischer Sterbeerzählungen ${ }^{3}$. Zunächst wird in dem Roman schlicht viel gestorben; er liefert - wie auch andere Texte Lewitscharoffs - reichlich Material für entsprechende Studien. Darüber hinaus ist Blumenberg aber auch von Interesse, weil das Erzählen vom Sterben auch ausdrücklich zur Debatte steht; der Erzähler erzählt vom Sterben, aber auch vom Erzählen vom Sterben. Wenn die genannten beiden Eigenschaften zweifellos in verschiedensten anderen Texten der Gegenwartsliteratur zu finden sind, ${ }^{4}$ so bietet Lewitscharoffs Roman schließlich auch etwas, was zumindest im E-Spektrum der aktuellen Literatur nicht ohne weiteres zu finden ist: eine relativ ausführliche Erzählung von Räumen jenseits der Todesschwelle.

Der folgenden Überlegungen können und wollen diese drei Aspekte weder für sich noch in ihrem Zusammenspiel ausführlich erläutern. Das Interesse richtet sich vielmehr auf einen formalen

\footnotetext{
1 Sibylle Lewitscharoff, Mit den Toten sprechen, in: dies., Vom Guten, Wahren und Schönen. Frankfurter und Zürcher Poetikvorlesungen, Berlin 2012, 115-133, 122.

2 Sibylle Lewitscharoff, Blumenberg. Roman, Berlin 2011 (Nachweise im Folgenden in Haupttext).

${ }^{3}$ Ich verzichte im gegebenen Zusammenhang auf eine Erläuterung dieses bestimmungsbedürftigen Begriffs.Vgl. aber Vf., Seinen Tod sterben. Kurt Martis exemplarische Sterbeerzählung Neapel sehen (1960), in: Pierre Bühler/Andreas Mauz, Grenzverkehr. Beiträge zum Werk Kurt Martis, Göttingen 2016, 225-249.

4 Vgl. etwa Heft 3/2015 der Zeitschrift für Germanistik: An der Grenze. Sterben und Tod in der Gegenwartsliteratur (hg. v. Anna Katharina Neufeld/Ulrike Vedder).
} 
Aspekt, der an einer der Sterbeerzählungen des Romans exemplarisch herausgearbeitet werden kann: Die Darstellungen des Lebensendes der Selbstmörderin Isa geben Anlass, auf die Faktur und die Leistung eines wiederholten Erzählens von einem Todesfall zu achten. Die Aufmerksamkeit gilt einem Fall des repetitiven Erzählens, der durch seinen Gegenstand identifiziert wird. Diese spezifische Fragerichtung gibt zugleich einen Eindruck davon, was es für die Autorin konkret bedeutet, wenn sie in einem poetologischen Essay bemerkt (man beachte den Superlativ), es sei »die vornehmste Aufgabe der Literatur, Totenwache zu halten und Totengespräche zu führen «. ${ }^{5}$ Zunächst ist aber in gebotener Kürze anzudeuten, mit welchen Zusammenhängen man es in Blumenberg zu tun bekommt.

\section{Löwenbesuch}

Lewitscharoffs elaborierter Roman stößt sich ab an der Rea-lität. Blumenberg handelt von Blumenberg, nämlich Hans Blumenberg (1920-1996), dem eigenwilligen Münsteraner Philosophen aus der Schule Joachim Ritters. Das unerhörte Ereignis, das die Erzählbewegung aus sich heraussetzt: Blumenberg erhält im fortgeschrittenen Alter Besuch von einem Löwen. "Der Löwe war da. Habhaft, fellhaft, gelb. [...] \Der Löwe ist zu mir gekommen, weil ich der letzte Philosoph bin, der ihn zu würdigen verstehtı, dachte Blumenberg." $(10 f .)^{6}$ Gesehen wird der Löwe, der dem Philosophen in seiner Schreibstube Gesellschaft leistet, ihn aber auch in den Hörsaal begleitet, allerdings nur von Blumenberg selbst und von einer zentralen Nebenfigur, der Nonne Käthe Mehliss. Die Handlung des Romans besteht dann in der Darstellung des Alltags des Philosophen, der in den Bann des Löwen geraten ist, bzw. den Ereignissen innerhalb eines Kreises Studierender - Gerhard, Hansi, Isa, Richard -, die in den Bann Blumenbergs geraten sind. Die Leben dieser Figuren werden alle auserzählt, bis in ihren Tod - und über diesen hinaus. So bietet

\footnotetext{
5 Aus der neueren Forschungsliteratur zur Autorin sticht das Text + Kritik-Heft heraus, Nr. 204,VII/2014 (Göttingen 2014). Zum hier interessierenden Themenfeld insbesondere Karl-Heinz Otts Aufsatz Sterben lernen. Das Naheliegendste sind für Sibylle Lewitscharoff die letzten Dinge (ebd., 14-24).

6 Ein Umstand, der auch für den historischen Blumenberg zutrifft.Vgl. ders., Löwen, Frankfurt a.M. 2001. Ich verzichte im Folgenden auf Hinweise zu den vielfältigen Beziehungen, die gerade auch von der Sterbethematik, wie sie im Buch zur Geltung kommt, zum Werk Blumenbergs bestehen. Siehe aber: Oliver Müller, Trost durch Weltbenennung. Realismusmüdigkeit und Transzendenzgewinn bei Sibylle Lewitscharoff, in:Text + Kritik 204, 63-73.
} 
der Roman nicht gerade ein Panoptikum, aber doch ein weites Spektrum an Sterbeszenen, nicht zuletzt eine Varietät gewaltsamer Tode. Die intensivste erzählerische Bearbeitung findet aber fraglos das Lebensende Isas. Um die Eigenart dieser Bearbeitung kenntlich zu machen, ist ein Exkurs nötig zum narratologischen Verständnis von Frequenz.

\section{Frequenz, narratologisch ${ }^{7}$}

Wenn jede reflexive Wahrnehmung einer Erzählung von der Basisdifferenz von Geschichte und Diskurs ausgehen kann - dem Was der Erzählung und ihrem Wie-, so kommt innerhalb der verschiedenen Diskursaspekte dem der Zeitgestaltung eine ganz zentrale Rolle zu. Während auf der Ebene des Geschichte in der Regel eine relativ klare Chronologie besteht, so bezieht insbesondere das literarische Erzählen seinen Reiz aus einer Umgestaltung dieser natürlichen Ordnung: Es wird etwa zuerst erzählt, wie der Flüchtling seinen Verfolgern zu entkommen sucht. Erst dann wird durch Rückblenden nachgereicht, weshalb es überhaupt zu Flucht und Verfolgung gekommen ist. Die unterschiedlichen Möglichkeiten, Ereignisse fiktiver oder faktualer Welten erzählerisch zu repräsentieren, erschöpfen sich allerdings nicht in solchen mehr oder minder komplexen Umstellungen der diskursiven Ordnung. Erzählungen gewinnen ihr erzählpraktisches Profil auch durch das Erzähltempo, die Beschleunigung oderVerlangsamung der narrativen Repräsentation. So können die Ereignisse eines Tages auf Romanlänge ausgedehnt werden; es können im Verlauf eines einzigen Satzes aber auch Millionen von Jahren vergehen. Konstitutiv für den Zeit-Diskurs von Erzählungen ist schließlich der Aspekt der Frequenz, das heißt die Häufigkeit eines Ereignisses in der Dimension der Geschichte im Verhältnis zur Häufigkeit der diskursiven Repräsentation dieses Ereignisses. Dieses Verhältnis kennt drei Grundtypen: das singulative Erzählen (ein Ereignis wird genau so oft dargestellt, wie es sich innerhalb der erzählten Welt ereignet), das repetitive Erzählen (ein Ereignis wird öfter erzählt als es sich in der erzählten Welt zuträgt) und das iterative Erzählen

\footnotetext{
7 Der folgende Abschnitt bietet narratologisches Allgemeingut. Für eine besonders klare Explikation der erwähnten Differenzen: Tilman Köppe/Tom Kindt. Erzähltheorie. Eine Einfuihrung, Stuttgart 2014, 180-191.
} 
(ein Ereignis, das sich in der erzählten Welt mehrfach zuträgt, wird weniger oft oder nur ein einziges Mal erzählt) ${ }^{8}$.

Der Reiz des repetitiven Modus liegt auf der Hand: Das betreffende Ereignis kann durch seine wiederholte Repräsentation aus verschiedenen Perspektiven gezeigt werden, und in vielen Fällen wird es das auch, sei es - um nur zwei Grundtypen zu nennen - gekoppelt mit einem Wechsel der Referenzinstanz (eine andere Figur sieht das gleiche Ereignis) oder auch nicht (die gleiche Figur nimmt das gleiche Ereignis etwa aus größerer zeitlicher Distanz erneut in den Blick).

Die genannten drei Frequenzmodi als solche vor Augen zu haben, ist von Bedeutung, um wahrzunehmen, dass sie einem Erzähler nicht immer in gleicher Weise zur Verfügung stehen; sie bilden keine permanente Option innerhalb der Palette der diskursiven Mittel. Der Grund für die konkrete Unerschwinglichkeit, die für das Folgende von Interesse ist, liegt im Allgemeinbegriff des Ereignisses. Denn als solcher verschleiert er, dass es unterschiedlichste Ereignisklassen gibt. Und die Klasse, die im gegebenen Zusammenhang interessiert, ist diejenige der - nach lebensweltlichem Maßstab - einmaligen Ereignisse. In ihrem Fall scheint die orientierende Leistung der Frequenzanalyse gerade zu versagen. Das Modell geht ja von einer prinzipiellen Wiederholbarkeit von Ereignissen aus. ${ }^{9}$ Diese Wiederholbarkeit ist aber bei fundamentalen lebensweltlichen Vorkommnisen gerade nicht gegeben: Menschen werden nur ein einziges Mal geboren, Menschen sterben auch nur ein einziges Mal. Ein Satz wie »Lange Zeit bin ich früh schlafen gegangen« lässt sich durchaus auf Lebensanfang und Lebensende übertragen - »Lange Zeit bin ich früh geboren worden/früh gestorben«. Der Reiz des übertragenen Satzes liegt dann aber gerade im Verstoß gegen unseren Realitätssinn; der Satz formuliert, mit Ryle gesprochen, eine "Kategorienverwechslung « ${ }^{10}$, nämlich eine »Anwendung von Prädikaten auf solche Gegenstände, die nicht zum Bereich der sinnvollen Verwendung dieser Prädikate

\footnotetext{
8 Das Schulbuchbeispiel für diese Frequenzgestaltung liefert der erste Satz von Prousts Recherche: „Longtemps je me suis levé de bonne heure."Diese Beschreibung des iterativen Modus, die als Umkehrung des repetitiven erscheint, ist allerdings nur prima vista plausibel. Wie etwa auch Kindt/Köppe einschärfen (Erzähltheorie, 191), liegt die Wiederholung in strengen Sinn nicht auf der Ebene des Ereignisses selbst, das phänomenologisch gesehen gar nicht wiederholbar ist (jedes frühe Aufstehen hat seine Individualität), sondern auf der Ebene eines Ereignistyps.

9 Auch dann, wenn man die in der letzten Anmerkung erwähnte Präzisierung von "Ereignis« zu »Ereignistyp» mitdenkt.

${ }^{10}$ Gilbert Ryle, Der Begriff des Geistes [1949], Stuttgart 2015, 14-24.
} 
gehören «" ${ }^{11}$ (Carnap: "Dieser Stein denkt an Wien«; Pap: "Sokrates ist eine Primzahl«). Aus derartigen Kategorienverwechslungen lässt sich literarisch, und damit intendiert, enormes Kapital schlagen. Die Literatur nimmt sich gern das Privileg, eine Figur mehrfach zur Welt kommen oder auch wiederholt sterben zu lassen; ${ }^{12}$ sie lässt auch Ich-Erzähler über ihren Tod hinaus weitererzählen, ${ }^{13}$ etc.

Wenn also der Frequenzmodus der Iteration für srealistischer Erzählungen im Fall der Ereignisse von Lebensbeginn und -ende nicht in Frage kommt, so finden diese, nebst dem etwas steifen singulativen Erzählen, im repetitiven Erzählen ihren genuinen Modus. Das eine und unwiederholbare Ereignis kann durch erzählerische Mehrfachbehandlung entfaltet werden - was gerade seine Einzigartigkeit und Unwiderruflichkeit illustriert. In diese Richtung scheint wenigstens das Exempel zu zeigen, das nun zu diskutieren ist.

\section{Isas Suizid}

Elisabeth Kurz, genannt Isa, gehört zum erwähnten Kreis von Blumenberg-Studierenden. Und sie ist ihm, der sie kaum bemerkt, in sehr ungesunder Weise verfallen. Der Grad ihrer intellektuellen wie amourösen Obsession mit dem akademischen Lehrer ist unter anderem abzulesen an einer imaginierten Liebschaft, die in einen Doppelsuizid mündet; er zeigt sich aber auch am missglückenden Versuch, Blumenberg ihre Liebe einzugestehen mittels eines Blumenstraußes und eines anonymen Schreibens. Tatsächlich begeht Isa dann auch Selbstmord, allein, indem sie sich von einer Brücke auf eine Autobahn stürzt. Dieses lebensweltlich nicht wiederholbare Ereignis wird nun eben wiederholt erzählt. Wie oft es erzählt wird, liegt allerdings nicht auf der Hand; die Zählung bemisst sich daran, was man noch als Erzählung und nicht nur Erwähnung dieses Ereignisses zu sehen bereit ist. Die folgenden Abschnitte konzentrieren

\footnotetext{
${ }^{11}$ Frank Kannetzky, Art. Kategorienfehler, in: Hans Jörg Sandkühler (Hg.), Enzyklopädie der Philosophie, Bd. 2, Hamburg 22010, 201-205, 201.

12 Jenny Erpenbeck hat letzteres unlängst mit ebenso großem Geschick wie Erfolg erprobt in Aller Tage Abend (München 2012). Der Roman zeigt nicht weniger als fünf denkbare Lebenswege bzw. Todes derselben Figur - ausgestreckt zwischen einem frühen Kindstod und einem späten Tod in Demenz.Vgl. Monika Hirt, Eine kleine Eschatologie? Betrachtungen zum Roman Aller Tage Abend von Jenny Erpenbeck, in: Hermeneutische Blätter 2016/1 (Für immer), Zürich 2016, 49-60.

${ }^{13}$ Vgl. Arto Paasilinna, Im Jenseits ist die Hölle los. Roman [1992], Bergisch Gladbach 2004 .
} 
sich auf vier Erzählgänge; sie sollen jeweils in ihrer Spezifik wie in ihrem Zusammenhang zur Geltung gebracht werden.

\section{Die erste Erzählung: von oben}

Die Erzählung von Isas Suizid, die das Ereignis in der erzählten Welt installiert, zeichnet sich aus durch einen direkten Fokus auf die Sterbewillige. Im Kapitel mit dem - zunächst - kryptischen Titel Nr. 255431800 zeigt ein heterodiegetischer Erzähler (ein Erzähler, der selbst nicht der erzählten Welt angehört) die Figur in den letzten Stunden vor ihrer Tat. Da dieser Erzähler typologisch gesehen allwissend ist (Genette spricht in diesem Fall, genauer, von "Nullfokalisierung $)^{14}$, hat er die Möglichkeit, Einblick zu nehmen bzw. geben in Isas psychisches Innenleben. Wie der gleich zu zitierende Auftakt des Kapitels zeigt, werden ihre mentalen Prozesse durch deren erzählerische Darstellung nur schwach überformt. Der Erzähler scheint, wo er die Figur zu Wort kommen lässt, nah an ihrer Wortwahl, ihrem Stil etc. zu bleiben; spricht er dagegen etwa von "mädchenhaften Riemchenschuhen" wird seine eigene Wahrnehmungsdisposition recht deutlich. Und dieses Nebeneinander scheint wichtig, da es im Verlauf des Kapitel wiederholt auftritt, allerdings ohne dass in jeden Fall deutlich würde, wer hier in dieser Weise spricht bzw. sprechen lässt.

"Gegen 15 Uhr zog sich Isa weiß an. Ein langes, fließendes Kreppkleid, weiße Kniestrümpfe und mädchenhafte Riemchenschuhe aus durchbrochenem Leder, die sie vor Jahren gekauft und nie getragen hatte. Sie stellte sich damit vor den Spiegel und kam sich absurd vor. Wie eine Debütantin mit weißer Perlenkette, jedenfalls nicht wie Patti Smith. [...] Sie zog den Mund kraus. Wie ein ironischer Schnörkel, dieser Mund, sagte sie sich, war aber sogleich von sich hingerissen. Ihre Augen so tief, tief, tief. Die Haut so weiß. Alles so weiß. [...] War sie nicht eine Wundermaschine? Ein nervtötend fades Leben hatte die Wundermaschine bisher führen müssen, nein, nicht nervtötend, sondern flau dahingleitend in ewigem Halbschlaf, warum so flau, konnte ihr einer sagen, warum, aber es war hinter ihr zerstäubt, diese flaue, fade Leben. Und was kam jetzt? Etwas Großes, Blasses, Bereitwilliges, sie nahm ein Papierschirmchen in die Hand, das auf einem Stück Cocktailmelone gesteckt hatte, öffnete es, schloß es, öffnete es." (73)

${ }^{14}$ Gérard Genette, Die Erzählung, München 21998, 134-138; 241-247. 
$\mathrm{Zu}$ diesem Ambiente, das Isa bald darauf in Richtung Autobahnbrücke verlassen wird, gehört ganz zentral auch ein Soundtrack:»The River ertönte jetzt schon zum zweiundzwanzigsten Mal, laut, viel lauter als sonst." (74) Bruce Springsteens klassischer Song liefert trotz der deutlich anders gelagerten Szenerie den stimmigen Ausdruck für Isas Befindlichkeit.

"Springsteen ging aufWasserfahrt, down to the river, Springsteen kostete das down mit Inbrunst aus, röhrte vom ärmlichen jungen Paar, dem kein Glück beschieden war [...]. Natürlich war Blumenberg kein Bauarbeiter, der für die Johnstown Company arbeitete, seine Konstruktionsarbeiten spielten sich auf dem Papier ab, aber der brennende Blumenbergblick wurde durch Springsteens Stimme lebendig, seine Augen starrten sie an." (74)

Dass Isa gerade in diesem Stück eine musikalische Resonanz auf ihre Situation findet, scheint psychologisch plausibel. Da das schwierige Schicksal der Liebenden im Liedtext aus der Perspektive des Mannes dargestellt wird - er ist das "I " des Stücks -, spricht er gleichsam als besserer Stellvertreter Blumenbergs, von dem Isa so gern ein »We«Wort der Liebe hörte.

Nachdem sich Isa mit ihrem Rad auf den Weg gemacht und Springsteens Song sich verflüchtigt hat, stellt sich in ihrem Sinn dann tatsächlich auch Blumenbergs Stimme ein - allerdings nicht mit einem bekenntnishaften »We«, sondern, vermittelt über einen philosophischen Gedanken, mit einem dunklen Imperativ. Blumenberg wird hier ausdrücklich zur Instanz, die sie zur Tat nötigt:

"[S]ie war der lebendige Gegensatz zur Welt und auf der Flucht, auch Fliehen ist Handeln, und Blumenberg - Blumenberg hatte ihr diesen Gegensatz angesonnen, Blumenberg hatte ihr Bescheid gestoßen, er schob sie von hinten an mit seinem langen, ellenlangen Zeigefinger, den er ihr in den Rücken gebohrt hatte und mit dem er bis an ihr Herz vorgedrungen war, fahr, fahr, fahr voran, mein armes durchtränktes Seelchen, fahr, du schlägst noch mit den Flügeln und erhitzt dich im weißen Kleid, bräutlich gestimmt nach Art der Engel, nicht der Menschen.« (78)

Schließlich auf der Brücke angekommen, wird der Gang der Erzählung noch einmal stark retardiert. Die Schilderung der konkreten Situation bzw. der inneren Befindlichkeit Isas fällt wesentlich kürzer aus als diejenige des "Perlentäschchens", das sie bei sich trägt, um es dann im Fahrradkorb zurückzulassen. Dessen Herkunft und Inhalt werden sehr genau beschrieben, wobei die Beschreibung des Täsch- 
cheninhalts - anders als die seiner Herkunft - nicht dem Erzähler zugeordnet ist, sondern in Gestalt eines eigentümlichen Dialogs erfolgt. Dieses Gespräch (s. u.) lässt sich sowohl als Selbstgespräch Isas auslegen, als auch, konkreter, als imaginiertes emotionales Gespräch Isas mit Blumenberg. Diese konkrete Lesart wird allenfalls dadurch gestützt, dass sich das Täschchen qua Form ganz direkt mit ihrer enttäuschten Liebe verbindet:

»Mein Geschlechtstäschchen, sagte sie laut und kam darüber ins Lächeln, sogar mit rotem Innenfutter.

Was genau war denn im Geschlechtstäschchen drin? Hm? Ein lauer Wind fuhr in ihre dünnen Haare. Sie grübelte mit zusammengezogenen Brauen wie ein Kind, das Fünf und Sieben zusammenzählen muß, während die Hände das Brückengeländer umklammerten und das rechte Bein auf die andere Seite schwang: Ein Hundermarkschein ist drin, Dummkopf, das mußt du doch wissen, in der kleinen Börse mit dem Druckknopf, der nicht richtig schließt, bißchen Kleingeld [...], und was ist mit dem Tempotaschentuch, hm? wozu braucht's denn ein Tempotaschentuch? [...] den Personalausweis, natürlich, kleiner Dummkopf $[\ldots]$, den Personalausweis hat ein ordentliches Schwabenkind immer dabei, ausgestellt in Heilbronn mit einem Bild, auf dem das Persönchen in Schwarzweiß aussieht, als wär's bös auf die Welt, aber was, um Gotteswillen, was will jetzt der Spatz auf dem Geländer, der so lustig mit dem Kopf ruckt, der - da war das linke Bein hinübergeschwungen, waren die Hände gelöst, und sie flog auch schon, flog engelgleich - « (79 f.)

Diese Sätze bilden den Abschluss des vorletzten Abschnitts des Kapitels. Der Wechsel zum letzten Abschnitt bedeutet dann eine starke stilistische bzw. erzählpraktische Zäsur. Hier schaltet sich erneut der Erzähler ein, der kühl reflektierend die letzten Lebenssekunden Isas rapportiert:

"Wenn immer behauptet wird, in den letzten Sekunden zöge das Leben in rasendem Lauf vorbei, so trifft das in diesem Fall nicht zu. Isa dachte an ihren Augenbrauenstift. Nicht an Blumenberg, wie es doch zu erwarten gewesen wäre. Bestimmt hatte sich die Kappe wieder gelöst und der Stift das Innenfutter verschmiert; sie flog und dachte mit aller Gewalt an die Schmiererei, biß die Zähne zusammen, fest, daß vom oberen linken Schneidezahn ein Stück absplitterte. Eine zu vernachlässigende Größe gemessen daran, was alles splitterte und brach, als der Körper auf dem Asphalt aufschlug." (80) 


\section{Die zweite Erzählung: kokette Poetik}

Im unmittelbar folgenden Kapitel ist erneut von Isas Suizid die Rede, allerdings in einem ganz anderen Register, nämlich in einem - so die Überschrift - Kurze[n] Zwischenstück darüber, wo die Zuständigkeit des Erzählers endet. Dieser Registerwechsel, dieser Übergang vom Erzählen zum Erzählen vom Erzählen, bringt das eben geschilderte Ereignis auf große Distanz. Die Selbstmörderin, diese Selbstmörderin, wird dem Erzähler zum Anlass einer recht koketten Reflexion über seine eigenen Wissens- und Darstellungsmöglichkeiten und konkreten Darstellungspräferenzen:

„Was weiß ein Erzähler, was weiß er nicht? Ob der Erzähler wirklich wissen kann, was einem Selbstmörder zuletzt in den Sinn kommt, ist fraglich. Natürlich, welche Kleidungsstücke getragen wurden, wie die Verlassenschaft aussah, welchen Anblick die Leiche bot, wie nah- und fernstehende Menschen darauf reagierten, das alles hat der Erzähler bis ins kleinste Details bei sich vermerkt; er braucht nur geschickt zu wählen, geschickt auszulassen, und er darf dabei nicht allzu streberhaft sein Aufzählungsmaschinchen in Gebrauch nehmen (unter einem Riesenhaufen an Kleinigkeiten wird sonst unmerklich und ohne Gefühlsverhaftung weggestorben). Gesetzt den Fall, all dies sei berücksichtigt und mit dem nötigen Schwung versehen: siehe da, vor den Augen des mitfühlenden Lesers löscht sich ein Buchstabenleben selbst aus." (81)

Diese kleine Poetik der Sterbeerzählung kann kokett genannt werden, weil sie mit wenigen Sätzen in den Strudel der Fiktionstheorie eintritt. Denn was soll hier gemeint sein mit einem "Wissen« oder gar "wirklichen" Wissen des Erzählers? Der Erzähler, auch der Erzähler, der sein Erzählen ausdrücklich reflektiert, weiß genau das, was ihn der Autor wissen lässt. Was er, als innertextuelle Instanz, ist und weiß, ist und weiß er von Gnaden der außertextuellen Instanz des Autors - so sehr er sich auch als Autorität zu etablieren bzw. demontieren sucht. ${ }^{15}$ Ihn, den Erzähler, auf ein Kriterium wie "Ehrlichkeit» behaften zu wollen, gleicht dem Eintritt in ein Spiegelkabinett. Und der Erzähler dieses Zwischenstücks tritt in Gestalt einer Unehrlichkeitsunterstellung auch beherzt in dieses ein: "Mit welcher Überlegenheit auch immer der Erzähler vorgibt, Bescheid $\mathrm{zu}$ wissen, er fischt hier bloß Luft aus der Luft. Wenn er ehrlich wäre, müßte er passen.« (82)

${ }^{15}$ Vgl. kritisch einführend etwa Kindt/Köppe, Erzähltheorie, 73-96. 
Vor diesem Hintergrund ist es aufschlussreich zu hören, wie sich der Erzähler den Fall zurechtlegt, der ihn zu seiner Übung in Selbstreflexion allererst motiviert:

"Der Fall Isa scheint zunächst klar. Wir haben es mit einer Verliebten zu tun, die sich im Irrealis verfangen hat. Eine riesige erotische Sehnsuchtswolke trägt die schmale Person mit sich herum, geschwellt von unerfüllbaren Wünschen, die bis in den Himmel hinaufaufsteigen; auf ihr tyrannisches Geheiß springt sie von der Brücke. So weit, so plausibel. Die Verfinsterung treibt und bläht den Menschen, überstäubt ihn mit falschem Zucker, bis kein Genuß in der Wirklichkeit mehr möglich ist." (82)

An diesem Punkt nimmt der nachdenklich tändelnde Erzähler das "zunächst" des erstzitierten Satzes auf und überführt die saubere Herleitung in eine Fragereihe, die sein Nichtwissen breit exponiert:

"Aber krallt sich die Wirklichkeit nicht jäh an den todbereiten Menschen an, genau in dem Augenblick, in dem es zu spät ist? Wenn eine besonnene Rückkehr nicht mehr möglich ist? Und als was blitzt das Wirkliche auf und strahlt in unwahrscheinlichem Glanz? Kleinklein, als Inhalt eines Krimskramstäschchens? Oder anders, als Spatz, in dessen ruckhaftem Hupf und Köpfchenwenden Heiterkeit und Anmut geborgen sind, daß man sich eigentlich bloß an den Haaren fassen und vergnügt loskichern müßte? [...] Dem Erzähler ist jedenfalls die Idee lieb und teuer, daß der Selbstmörder im letzten Moment von der Wirklichkeit verspottet wird, die er so verschmäht und vernachläßigt hat. Wie weggeblasen, all der falsche Zauber." (82f.)

Die Koketterie des Erzählers setzt sich fort, wenn er diese Präferenz für einen spöttischen Einspruch der Wirklichkeit (hier Gestalt gewinnend im schmierenden Augenbrauenstift) bescheiden glossiert durch den Verweis auf einen der basalsten Sachverhalte literarischer Kommunikation: die "naturgemäße A Autonomie des Lesers. Und der autonome Leser, den sich der Erzähler zurechtlegt, vertritt eben eine andere, eine romantische Position: "Dem Leser steht es naturgemäß frei, zu denken, was er will. Im Fall Isas mag er glauben, sie sei dem Phantom Blumenberg entgegengesprungen und kein einziger Wirklichkeitsschnipsel hab sich mehr zwischeneindrängen können. Bittesehr, dem Leser darf nicht widersprochen werden.« (83)

Für den hier diskutierten Aspekt des repetitiven Erzählens vom Sterbens scheint es wichtig, dass der Erzähler in den zitierten Erwägungen stark an den Ursachen der Selbsttötung interessiert ist. Sie 
müssen offensichtlich zur Darstellung kommen, und im gegebenen Fall seien sie ja auch erfreulich "plausibel" (82). Interessanterweise gibt sich der Erzähler an dieser Stelle aber mit einer strukturellen Antwort zufrieden. Der Suizid wird plausibel durch die "Sehnsuchtswolke» und das "tyrranische Geheiß« ihrer Wünsche. Die konkrete zwischenmenschliche Beziehung, die in der Lage ist, diese mächtige Wolke zu erzeugen, spielt keine Rolle. Was, anders gesagt, außen vor bleibt, ist das grelle Licht, das durch Isas Tod auf den Protagonisten des Romans fällt: Blumenberg.

Diese im Kurzen Zwischenstück angeschlagene Repetitions- und Reflexionsstufe hat nun einen erheblichen Effekt. Sie kommentiert nicht nur retrospektiv die erste Erzählung, sie wirkt auch prospektiv als Vorschein auf alle folgenden Erzählungen vom Suizid. Sie ist eine ausdrückliche Einladung dazu, dem Erzähler bei seinem Handwerk auf die Finger zu sehen.

\section{Die dritte Erzählung: von unten}

Die dritte Erzählung, die sich zu Beginn des Kapitels Fragwürdiger Engelbescheid findet, nimmt den Erzählfaden an genau der Stelle wieder auf, wo er am Ende des vorletzten Kapitels verlassen wurde. Der punktgenaue zeitliche Anschluss verbindet sich allerdings mit einem Wechsel des Schauplatzes; der Erzähler nimmt das Ereignis nun folgerichtig von unten in den Blick:

"Vollbremsung. Tohuwabohu, ein Wunder, daß nicht gleich mehrere Fahrzeuge ineinanderkrachten. Polizei und Notdienst waren rasch zur Stelle. Was die Sanitäter an blutigen Kleidungsresten, Fleisch, zermalmten Knochen vom Asphalt kratzten, war nicht mehr als Person zu erkennen. Der Lastwagen der Firma Zapf war mit mehreren Rädern darüber gerollt, hatte Teile mitgeschleppt, bis er hinter der Brücke zum Stehen gekommen war. Der Familie, falls es eine gab, würde der Anblick dieser Reste nicht zuzumuten sein. Dennoch ließ sich die Person, um die es sich wahrscheinlich handelte, identifizieren.

Polizisten fanden ein weißes Täschchen im Korb des auf der Brücke abgestellten Fahrrades [...], mit hundertsechs Mark und vierzig Pfennigen darin, einem Lippenstift, [...] keinem Schlüssel, aber einem Personalausweis mit der Nummer 255431800, gültig bis 18.3.1984, ausgestellt auf den Namen Elisabeth Kurz, wohnhaft 7100 Heilbronn, Am Schafberg." (102) 
Isas Täschchen fungiert damit als materiales Bindeglied zwischen der ersten und der dritten Erzählung. Allerdings ist es durch die Weise, wie es in der ersten Erzählung zur Darstellung kommt, eben wesentlich mehr als ein Ding. Es stellt ein Vermächtnis dar, ein objekthaftes in einen seltsamen Dialog gemummtes sletztes Wort‘, auch wenn die Polizisten, anders als die Leser, nichts wissen von Isas letzter Sorge, die durch den Spott der Wirklichkeit eben nicht Blumenberg galt, sondern dem schmierenden Lippenstift. Die Polizisten sichten das Täschchen dagegen ausschließlich im Interesse einer Identifikation der Selbstmörderin. Und dieser amtlich-administrative Zugriff auf die Person, der an den Hintergründen, die eine junge Frau dazu veranlassen, in einem weißen Kleid von einer Autobahnbrücke zu springen, weniger interessiert ist als an einem lückenlosen Protokoll und einer möglichst frühzeitigen Normalisierung des Verkehrsflusses, kündigt sich bereits an, wenn die Nummer des Personalausweises über dem Kapitel steht, das Isas Liebes- und Lebensnot so plastisch zeigt wie kein anderes. Deutlich wird überdies, dass auch der Erzähler angesichts des zerschmetterten Körpers nicht seine relative Kälte verliert. Wenn er rapportiert, die Sanitäter würden die Leichenteile "vom Asphalt kratzen", so macht er unmissverständlich klar, nicht von der feinfühligen Sorte zu sein. Zugleich löst er mit dieser und weiteren Stellen ein, was in der poetologischen Einschaltung angekündigt wurde: dass er auch den "Anblick der Leiche" (81) einbeziehen wird in sein Kalkül der »Auswahl " bzw. "Auslassung".

Diese distanzierten Stimmen angesichts der Folgen von Isas Sprung werden folgerichtig abgelöst durch eine emotionalere, wenn sich der Bericht des Erzählers u. a. im Modus der direkten Figurenrede der Schockerfahrung des Fahrers zuwendet. Zugleich wird deutlich, dass Isas bewusste Inszenierung ihres Freitods, das Selbstbild des Engels "Alles so weiß« (73) - die gewünschte Wirkung erzielt.

"Weiß wie ein Engel, murmelte der Fahrer vor sich hin. Er schwitzte stark, hielt die Hände gefaltet, sie fuhren ihm auseinander, er wischte sich über die Stirn, faltete sie wieder. Er war keiner zusammenhängenden Aussage fähig. Ein drahtiger Bursche mit Zopf, den so leicht nichts erschüttern konnte. Jetzt zitterte ihm das rechte Bein. Ohne Vorwarnung, von oben, nicht einfach auf der Straße. Von oben, sagte er immer wieder [...]. Was Weißes." (102f.)

Die Darstellung richtet sich schließlich auch auf die Reaktion des Beifahrers Harry, der erneut einen anderen Umgang mit dem Ereignis zeigt, die Bierfahrer-Variante der Professionalität der Polizisten. 
Auf deren Ansage, der Lastwagen könne nicht weiterfahren, sie sollten in Münster übernachten, meint er: »Ich kann übernehmen. Bin total ruhig. Da zittert nix. Harry konnte seinen Ärger nicht länger unterdrücken. Jetzt steckten sie schon zwei gottverdammte Rattenstunden fest. Was müssen diese Arschlöcher sich umbringen und anderen den Tag damit versauen!« (104) Harry normalisiert das eben Geschehene, indem er, ungeachtet seiner Drastik, dessen Konsequenzen für die eigene Aufgabe betont und den Fall einordnet als Fall unter Fällen. Isa ist bloß ein weiteres "Arschloch", das sich für die Folgen seiner Tat für Dritte nicht interessiert.

\section{Die vierte Erzählung: Nahverhältnisse}

Auf diese dritte Erzählung, die zeitlich wie räumlich das Komplement zur ersten darstellt, folgt im gleichen Kapitel eine vierte. Diesmal liegt der Fokus auf zwei anderen Blumenbergianern, nämlich Richard und Gerhard, die sich im »Mauritztor» verabredet haben.

"Er [Richard] stand extra auf und fasste ihn [Gerhard] zur Begrüßung an der Schulter. Merkwürdig, Richard, war für gewöhnlich viel zu verdrossen für so eine Aktion, mit Begrüßungen trieb er keinerlei Aufwand. [...]

Weißt du es schon?

Gerhard wußte von nichts. [...]

Noch bevor das Bier kam, erzählte Richard. Er wusste ziemlich genau Bescheid, wo und wie und wann." (107)

Das Ereignis des Suizids einschließlich vieler Details sind an diesem Punkt bekannt. Nicht nur in der Kommunikation zwischen dem Text und dem Leser ist es daher möglich, die Zusammenhänge in verknappter Form anzusprechen - »es [...] wo und wie und wann" -, auch innerhalb der erzählten Welt führt »es« im Fall der Nahverhältnisse nicht zu weiteren Erläuterungen des Suizids selbst, sondern zu Bemühungen, dieses Ende mit früheren biographischen Episoden in Verbindung zu bringen. ${ }^{16}$ "Gerhard schien nicht versessen darauf, mit den unappetitlichen Details der Chose genauer bekannt zu werden." (109) Im Fall Richards, der einmal etwas mit Isa hatte (was Gerhard nicht weiß und auch nicht wissen soll), geht diese Kontinuitätsbildung eher in negative Richtung: »Daß sich Isa auf so über-

${ }^{16}$ Das betrifft insbesondere auch die hier nicht einbezogene Episode der Begegnung Gerhards mit Isas Eltern, denen Gerhard in ihrer Ratlosigkeit und Trauer beistehen soll (119): "Sagen Sie noch was, bitte hören Sie nicht auf zu erzählen. Das klang wie ein Flehen.» 
triebene Weise umgebracht hatte, passte zu ihr.Vielleicht.Vielleicht auch nicht. Daß sie sich hatte plattwalzen lassen, hatte ihn denn doch überrascht. In Richard rührte sich kein Mitleid [...]." (108f.) Was beide Freunde verbindet: der Konsens, die Ausnahmeneuigkeit klassisch männlich zu bearbeiten durch reichlichen Alkoholkonsum.

\section{Frequenzkalkül}

Ein und dasselbe Ereignis wiederholt zu erzählen scheint grundsätzlich besonders dann motiviert zu sein, wenn das Ereignis durch die mehrfache Bearbeitung gerade nicht dasselbe bleibt - wenn die Wiederholung eine Anreicherung des Dargestellten einschließt, welcher Art auch immer. Isas Suizid wäre offenkundig ein anderer, fehlte eine/fehlten mehrere der diskutierten Wiederaufnahmen. Besonders einschneidend wäre es wohl, beließe es die Autorin bei der zweiten Erzählung oder auch bei der ersten. >Fehlte im ersten Fall das Finale der slangen Perspektive - die intime Begegnung mit dem angeschlagenen psychischen Haushalt der Sterbewilligen und damit insbesondere die Einsicht in die ausgeprägte Inszenierung ihres letzten Akts, konterkariert durch den Spott der vernachlässigten Wirklichkeit, so sfehlte im zweiten die Verdichtung der skurzen< Perspektive, die Bereitschaft, den Aufschlag des Körpers einschließlich der zugehörigen administrativen und psychischen Vorgänge zu dokumentieren. Diese und alle weiteren knappen Erzählungen und Erwähnungen sind zusammengenommen das soziale Ereignis "Isas Suizid«.

$\mathrm{Zu}$ überprüfen wäre nun, ob Lewitscharoffs repetitives Erzählen von Isa allenfalls eine produktive Heuristik furr die Arbeit an anderen Suiziderzählungen liefern könnte. Es ist ja durchaus denkbar, dass sich die Eigenheiten der einzelnen Erzählungen, ausführlicher erschlossen, zu typologischen Unterscheidungen weiterentwickeln lassen. Dabei ginge es nicht nur um eine Aufmerksamkeit für Tätererzählungen, für unterschiedliche Formen von Zeugenerzählungen, Professionellenerzählungen, Familienerzählungen und Freundeserzählungen; zu prüfen wäre auch, ob diese Figuren und ihre emotionalen Responses und Copingstrategien mit bestimmten diskursiven Mitteln korrelieren. Konkret: Ist Lewitscharoffs Roman vielleicht auch für andere Texte repräsentativ, wenn die Reihe des Wiedererzählens gerade durch die Tätererzählung eröffnet wird? Folgt die Darstellung der letzten Stunden Isas allenfalls auch darin einem verbreiteten Muster, dass diese Darstellung - ins Unreine gespro- 
chen: die Selbstdeutung - nicht nur am Anfang steht, sondern auch deutlich ausführlicher ausfällt als die folgenden Fremddeutungen?

Einleitend wurde darauf verwiesen, dass die Identifikation einer Erzählung als Wiederholung einer Grunderzählung nicht auf der Hand liegt. Diese zentrale Problematik zeigt sich, schließlich, auch an einem entscheidenden Punkt, der bislang offen blieb. Denn die Hinweise zu den verschiedenen Erzählgängen werden ja wohl die Frage aufgeworfen haben, ob und wie allenfalls auch Blumenberg selbst in den Reigen des repetitiven Erzählens einbezogen ist. Er wird es - wenn man denn bereit ist, das in Frage stehende Ereignis auch als Anonymes zu anerkennen, wenn man zudem bereit ist, auch einen anonymen Vorschein als Modus von Wiederholung zu sehen. Denn die Autorin imaginiert nicht etwa ein späteres Nachdenken Blumenbergs über das traurige Ende seiner Studentin und seinen Anteil an diesem. Im Kapitel vor der Tätererzählung platziert sie ihn, tragisch-ahnungslos, in nächster Nähe zum Tatort:

»Seine zügige Fahrt wurde kurz vor Münster gebremst. Er geriet in einen Stau, Zorn wallte in ihm hoch. Daß ihm diese dumme Aufhaltung zusätzlich widerfahren mußte! [...] Langsam, wieder und wieder stockend, setzte sich die Kolonne in Bewegung. Etwas zügiger rollte Blumenberg am blaulichtumzuckten Ort des Geschehen vorüber, wo ein gelber Lastwagen hinter der Autobahnbrücke auf dem rechten Fahrstreifen stand. [...] Was für ein Unfall geschehen sein mochte, war nicht zu erkennen. Jedenfalls befand sich kein beschädigtes Auto an der Seite.«(70-72)

\section{Eschatologisches Erzählen: »Blumenberg!»}

Abgesehen von den Verbindungen, die sich von Blumenberg aus zu anderen Suiziderzählungen oder auch zur Funktionalität des repetitiven Erzählens etablieren ließen, wäre es zunächst schlicht fällig, die Arbeit am Roman weiter zu treiben. Vor allem wären die Erzählungen von Isas Suizid zu beziehen auf die vielen anderen Sterbeerzählungen: auf den Hirnschlag, den Gerhard unmittelbar im Anschluss an einen glänzenden Probevortrag für eine Professur ereilt, auf den Raubmord, dem Richard zum Opfer fällt, auf den Zusammenbruch des Poeten und Propheten Hansi nach einer irren Verkündigung am Bahnhof Zoo, auf Käthe Mehliss, die von ihren Mitschwestern behütet im hohen Alter stirbt (»Gleich geht's wieder los, ihr werdet sehen!«, 197) - und schließlich auch auf Blumenbergs Sterben, das, 
unerzählt, in einem Abschnittswechsel erfolgt: "Er wusste um die Kürze der Frist, die ihn noch gewährt blieb. [/] Am 28. März 1996 fand ihn seine Frau tot im Bett liegen. Eine Spur Löwengeruch hing im Zimmer, aber so gering, daß die Frau in ihrer Aufregung und der herbeigeholte Arzt nichts davon bemerkten.« (202)

Das Erstaunliche aber eben: Der Erzählfaden wird weiter und offensiv über die Grenze des Todes hinaus fortgesponnen. Die vielen Tode werden aufgehoben in ein Jenseits; die Toten, auch der Löwe, treffen sich wieder in einem Jenseitsraum, wo sie verdämmernd sich zunehmend abhanden oder ganz zu sich kommen. Der monumentale Schluss des Romans:

"Königlich, königlich schollernden Klanges fuhr Blumenberg! aus dem Rachen des Löwen. War der Mann in der Höhle bisher nicht viel mehr gewesen als Luft an der Luft, schien auf den Namenszuruf hin eine andere Materie ihn zu befuillen. Lichtsendendes Blut zirkulierte in seinen Adern. Er strahlte und zitterte und hielt die schwankenden Arme weit ausgebreitet. Da hieb ihm der Löwe die Pranke vor die Brust und riß ihn in eine andere Welt.» (216)

— Andreas Mauz ist geschäftsführender Oberassistent am Institut für Hermeneutik und Religionsphilosophie (IHR) der Theologischen Fakultät Zürich. 